\title{
Twin Peaks: An ERP Study of Action Planning and Control in Coacting Individuals
}

\author{
Natalie Sebanz ${ }^{1}$, Günther Knoblich ${ }^{1}$, Wolfgang Prinz ${ }^{2}$, \\ and Edmund Wascher ${ }^{3}$
}

\begin{abstract}
Previous studies have shown that perceiving another's actions activates corresponding representations in an observer's action system. The present study investigated how performing a task with another person affects action planning and control. Reaction times (RTs) and event-related potentials were measured while participants performed a go/no-go task alone and with another person. Three effects of acting together were observed. First, RTs were slowed when individuals had to respond
\end{abstract}

\section{INTRODUCTION}

Research in social cognitive neuroscience has made it clear that cognitive and neural processes underlying perception and action cannot be fully understood without taking into account how they are shaped by social context. A range of behavioral, neuroimaging, and neurophysiological studies has provided evidence that processes related to the planning, implementation, and performance of goal-directed actions are modulated by observing another's actions, suggesting that action perception and execution are tightly linked. These studies focused on social settings in which an individual-the participant - either merely observed another's actions or performed certain actions while concurrently perceiving actions. In the present study, we employed behavioral and electrophysiological techniques to investigate how the planning and control of actions are shaped by acting with another individual rather than by just observing another's actions. By replacing the usual observer-actor context with a setting in which two participants performed a task together, it was investigated whether another's actions become part of one's own action plan and whether acting in turns places special demands on action control.

\footnotetext{
${ }^{1}$ Rutgers University, Newark, ${ }^{2}$ Max Planck Institute for Human Cognitive and Brain Sciences, Munich, Germany, ${ }^{3}$ University of Dortmund, Germany
}

to a stimulus referring to the other's action, suggesting that an action selection conflict occurred. Second, at frontal sites, a stimulus referring to the other's action elicited a similar electrophysiological response as a stimulus referring to one's own action. Finally, on no-go trials, P300 amplitude was significantly larger in a group setting, indicating that an action was suppressed. These findings provide evidence that individuals acting in a social context form shared action representations.

\section{Observing Others' Actions}

What happens when we observe others' actions? According to ideomotor theories, observing (or imagining) a certain event in the environment activates representational structures involved in the planning and execution of the action that has been learned to produce this event (Jeannerod, 1999; Prinz, 1997; Greenwald, 1970). For example, hearing the sound of hands clapping should activate one's own representation of clapping. This, in turn, should facilitate or even trigger the performance of clapping movements. Behavioral and neurophysiological findings support the claim that perceiving an action activates corresponding motor representations in an observer and facilitates the performance of perceived action.

Studies on nonconscious mimicry provide evidence that observing an action creates a tendency to perform this action. In many social situations, individuals unintentionally adopt the postures, mannerisms, and facial expressions of an interaction partner (Chartrand \& Bargh, 1999). For example, individuals wiggle their foot more when in the presence of a heavy footwiggler. This phenomenon can be explained by a close link between perception and action, as assumed by ideomotor theories. When an action is perceived, a corresponding representation in the observer's motor system is activated, thus creating a tendency in the observer to perform this action (cf., Chartrand \& Bargh, 1999).

Neurophysiological and brain imaging studies have revealed common neural substrates for action observation and action execution. Mirror neurons in the ventral 
premotor cortex and the parietal cortex of macaque monkeys have been shown to discharge both when a monkey performs an action and when it observes another individual performing the same action (Rizzolatti \& Craighero, 2004; Rizzolatti, Fogassi, \& Gallese, 2001; Gallese, Fadiga, Fogassi, \& Rizzolatti, 1996; Rizzolatti, Fadiga, Gallese, \& Fogassi, 1996). Thus, they can be regarded as a possible neural substrate for the direct mapping of observed actions onto motor representations. Interestingly, mirror neurons discharge not only when an interaction with the object is directly perceived, but also when the action effect can be inferred (Umiltà et al., 2001) or perceived in a different sensory modality (Kohler et al., 2002). This suggests that the mirror system supports the mapping of action effect representations, rather than actual movements.

First, electrophysiological evidence that common structures underlie action perception and performance in humans was provided by electroencephalogram (EEG) studies on the reactivity of cerebral rhythms during movement observation (e.g., Gastaut \& Bert, 1954). These studies showed that the $m \mu$ rhythm, which is normally present during motor rest and disappears during active movements, was blocked during the observation of another person's movements (for recent replications, see Altschuler, 2000; Cochin, Barthelemy, Roux, \& Martineau, 1999). This finding suggests that observing an action performed by another individual is associated with brain activity similar to that when performing the action oneself. However, these findings cannot be taken to make any claims about localization. More recently, imaging studies have shown that several brain areas are activated when an action is imagined or carried out, as well as when it is observed in others (Buccino, Binkofski, \& Riggio, 2004; Grèzes, Armony, Rowe, \& Passingham, 2003; Blakemore \& Decety, 2001; Grèzes \& Decety, 2001; Hari et al., 1998; Decety, Grèzes, et al., 1997; Grafton, Arbib, Fadiga, \& Rizzolatti, 1996; Rizzolatti, Fadiga, Matelli, et al., 1996). It is assumed that a mirror system exists also in humans, with core regions comprising the rostral part of the parietal lobule, the lower part of the precentral gyrus, and the posterior part of the inferior frontal gyrus (Rizzolatti \& Craighero, 2004).

\section{Acting While Observing Others' Actions}

Given that observing an action activates corresponding representations in the observer's action system, how does this affect the performance of actions during observation? Behavioral studies have shown that performance of an action is facilitated when it is identical with a concurrently observed action, and is impaired when the observed action and the action to be performed do not match (cf., e.g., Kilner, Paulignan, \& Blakemore, 2003; Brass, Bekkering, \& Prinz, 2001; Stürmer, Aschersleben, \& Prinz, 2000). For example, performing a grasping movement takes less time when one observes a hand performing a grasping movement than when one observes a hand performing a spreading movement (Stürmer et al., 2000).

Such effects can be explained by the assumption that common representational structures are involved in action perception and action planning. Action planning comprises the preparation and implementation of goaldirected movements (see Hommel, 2006). It is facilitated when a representation of the action to be performed is already activated through perception (e.g., when one observes another person performing the action one is about to carry out). When a different action representation is activated through perception, an action selection conflict arises and must be resolved by mechanisms of action control. For example, this is the case when one observes a person lifting a finger while one is about to make the opposite movement (cf., Brass, Bekkering, et al., 2001).

\section{Acting with Others}

Action planning and control in a social context are not only modulated by action perception. In a series of studies, Sebanz, Knoblich, Stumpf, and Prinz (2005) and Sebanz, Knoblich, and Prinz (2003, 2005) showed that a stimulus associated with another's action can also activate a representation of the other's action, without the action actually being observed. In particular, individuals experienced an action selection conflict when they had to respond to a stimulus that was associated with the action of another person acting in the same social context. This suggests that they represented an action alternative at a coactor's disposal in a functionally similar way as to their own.

The aim of the present study was to investigate in more detail how acting together affects action planning and control. Through event-related potential (ERP) measurements, results from previous reaction time (RT) studies can be extended in two ways. First, by analyzing the electrophysiological response to stimuli referring to another's potential action, it is possible to gain a better understanding of the source of action selection conflicts. Second, action control processes occurring in the absence of overt action can be investigated. ERPs were measured while participants were performing a task alone (individual condition) and together (group condition; see Figure 1). By comparing specific ERP components in the two different settings, we determined whether a coactor's action alternative is taken into account during action planning, and whether holding back one's action when it is another's turn requires increased inhibitory control.

Participants performed an RT task. Two action alternatives, a left button press and a right button press, were distributed among them, so that each was responsible for one action alternative. On each trial, they were 


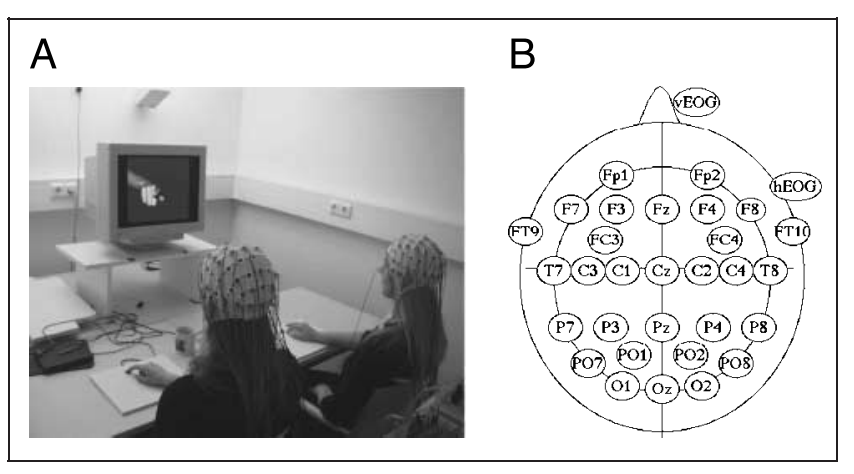

Figure 1. Simultaneous EEG measurement in two participants. (A) Experimental group setting. (B) A head icon with electrode sites. These were the same for the two participants in each pair.

presented with a picture of a hand. They were instructed to respond to the color of the ring on the index finger (see Figure 2). Each participant responded to one color (red or green) with a button press (go/no-go task). In the group condition (Figure 2A), one participant responded to red and the other responded to green. Thus, on each trial, one participant acted (go) whereas the other did not (no-go). For each participant, half of the trials comprised go trials and half were no-go trials (other acts). In the individual condition (Figure 2B), each participant performed the same task alone (e.g., responding only to red). Again, half of the trials were go trials and half were no-go trials (nobody acts).

The pointing direction of the hand was task-irrelevant. However, we systematically varied correspondence between pointing direction and the action to be performed (cf., Figure 2). On half of the trials, the irrelevant pointing direction corresponded to the spatial position of the required action (compatible trials; e.g., a left button press is required, and the finger points to the left). On the other half of the trials, the pointing direction did not correspond to the spatial position of the required action (incompatible trials; e.g., a left button press is required, but the finger points to the right). In other words, on compatible trials, the finger pointed toward the person who should respond, whereas on incompatible trials, it pointed toward the person who should not respond (group condition) or toward an empty seat (individual condition).

\section{Predictions}

\section{Action Planning}

Results from spatial compatibility studies have shown that when a person has two spatially arranged action alternatives at his or her disposal (e.g., a left button press and a right button press) and the (task-irrelevant) spatial dimension of the stimuli he or she responds to overlaps with the action alternatives (e.g., stimuli point- ing to the left or to the right), the task-irrelevant spatial information automatically activates a representation of the spatially corresponding action (Wascher, Reinhard, Wauschkuhn, \& Verleger, 1999; Kornblum, Hasbroucq, \& Osman, 1990; Simon, 1990). For example, when a stimulus points to the left, this will automatically activate a representation of the left action. Accordingly, RTs are faster when the response required by the relevant stimulus feature (e.g., color) corresponds to the action activated by the irrelevant spatial stimulus feature (compatible trials), and are slowed when two conflicting

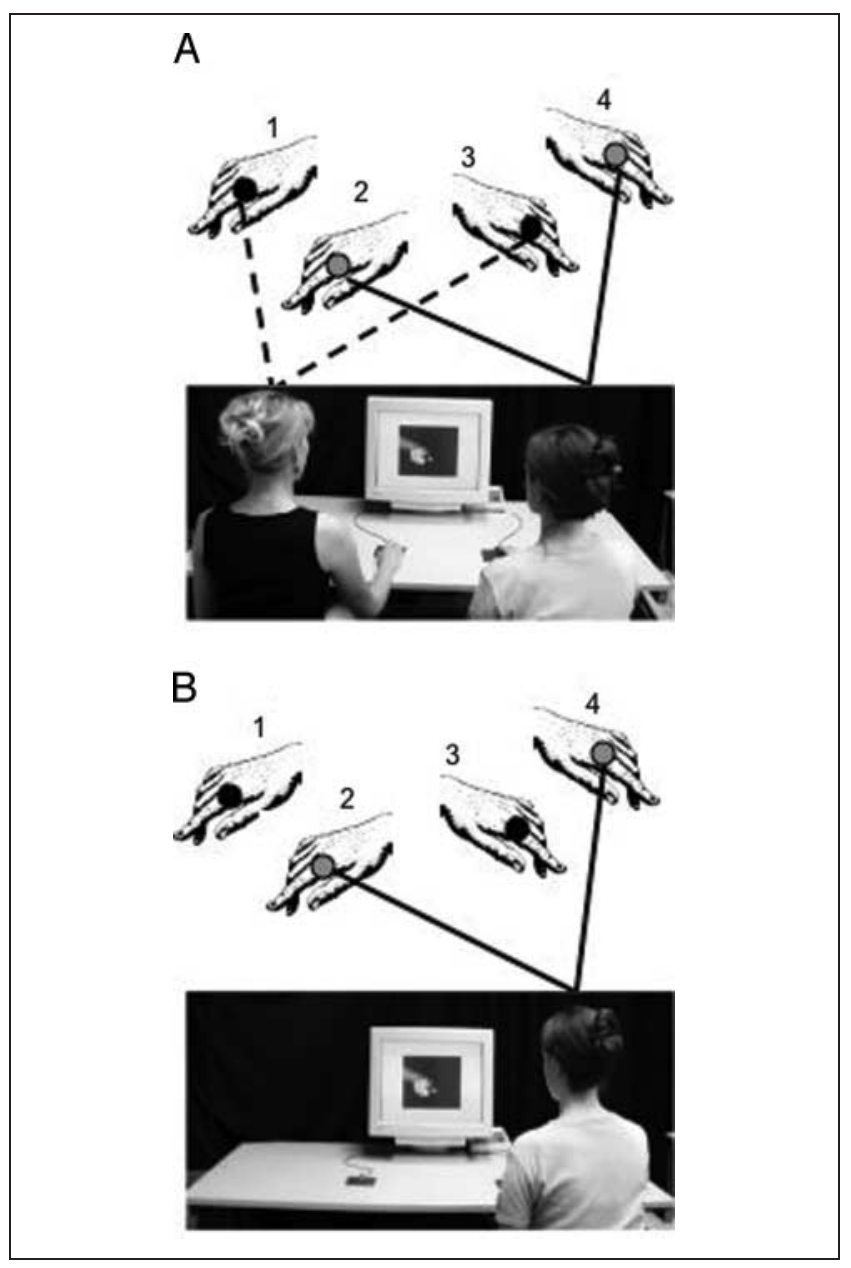

Figure 2. Individual and group conditions. Pictures of a hand pointing to the left or to the right with a colored ring on the index finger are shown. In the example illustrated here, (A) the left person is instructed to respond to red (dashed lines) and the right person is instructed to respond to green (solid lines). For the left person, the stimulus labeled " 1 " is compatible because the pointing direction of the hand corresponds to the spatial position of the action to be made in response to ring color (both left). The stimulus labeled " 3 " is incompatible because the pointing direction refers to the other action alternative (the left action should be performed, but the stimulus points to the right). (B) For the right person, the stimulus labeled " 4 " is compatible, and the stimulus labeled " 2 " is incompatible. Note that the numbers and lines are for illustrative purposes only and were not shown in the actual experiment. 
action representations are activated (incompatible trials). For example, a left button press is executed faster when the stimulus points to the left than when it points to the right, although the pointing direction is taskirrelevant. This spatial compatibility effect is usually not observed in go/no-go tasks where only a single action alternative is available (cf., Hommel, 1996).

We used this phenomenon to investigate whether individuals in the group condition represent the action alternative at their coactor's disposal in a way that is functionally equivalent to their own. In this case, the irrelevant pointing stimulus should automatically activate a representation of their own action when it corresponds to the spatial position of their own action alternative, and it should activate a representation of the other's action when it corresponds to the action alternative at the other's disposal (cf., Sebanz, Knoblich, \& Prinz, 2003). This should manifest itself as follows. First, in the group condition, RTs should be faster on compatible trials and slower on incompatible trials. In the individual condition, responses on compatible and incompatible trials should be equally fast because the pointing stimulus does not activate a competing action representation when it points away.

Second, ERP studies have shown that a specific electrophysiological component, the P300 (P3), is modulated by compatibility. When a two-choice RT task as described above is performed, the P3 amplitude peak is typically larger on compatible trials than on incompatible trials (Zhou, Zhang, Han, \& Tan, 2004; Valle-Inclán, 1996; Renault, Fiori, \& Giami, 1989; Ragot, 1984). This difference is assumed to reflect both perceptual interference (i.e., increased processing difficulty when attending to the left interferes with processing a right feature, and vice versa) and response selection conflict (Zhou et al., 2004; Valle-Inclán, 1996). More generally, the P3 is regarded as a component reflecting the evaluation of action-relevant stimuli (cf., Kok, 2001).

If the P3 were to reflect only perceptual interference, the typical pattern of a larger amplitude peak on compatible trials compared to incompatible trials should also appear in a go/no-go task, where participants are in charge of only one action alternative. The reasoning is that, if the effect arises as a consequence of perceptual processing, it should occur independent of whether a response selection conflict is present. Hence, it should not make a difference whether the go/no-go task is performed alone or together. Both in the group condition and in the individual condition, the P3 amplitude peak should be larger on compatible trials than on incompatible trials.

However, if one assumes that the P3 amplitude also reflects the evaluation of a stimulus with respect to action planning (Kok, 2001), one should expect a modulation of the P3 amplitude depending on whether the task is performed alone or together. In particular, it should make a difference whether one responds to a stimulus that refers to another's action (group condition, incompatible trials) or to a stimulus that does not refer to another's action (individual condition, incompatible trials). Therefore, we predicted a larger positivity on incompatible trials in the group condition compared to incompatible trials in the individual condition. Given the functional heterogeneity of the P3, the effects of perceptual interference and action-related stimulus evaluation are not mutually exclusive. Rather, we expected to find differences in terms of temporal and/or spatial distribution that would reveal the presence of two functionally dissociable effects.

\section{Action Control}

Acting in turns implies that one needs to withhold one's action when it is the other's turn. Does this require increased inhibitory control? According to ideomotor theory, the answer should be yes. When a stimulus requires an action from another person (no-go trial), a representation of this action will be activated, provided that the other's task is known (cf., Sebanz, Knoblich, \& Prinz, 2005). The emerging tendency to act must be suppressed when it is the other's turn. Thus, most likely, more inhibitory control is needed on no-go trials in the group condition, where an activated action representation must be suppressed, compared to the individual condition, where no action representation is activated on the no-go trials. This prediction can be tested by analyzing No-go P3. It is well-established that this component reflects action control (Fallgatter \& Strik, 1999; Jackson, Jackson, \& Roberts, 1999), and, in particular, response inhibition on no-go trials (Bokura, Yamaguchi, \& Kobayashi, 2001; Tekok-Kilic, Shucard, \& Shucard, 2001; Falkenstein, Koshlykova, Kiroj, Hoormann, \& Hohnsbein, 1995; Roberts, Rau, Lutzenberger, \& Birbaumer, 1994; Pfefferbaum, Ford, Weller, \& Kopell, 1985). If additional action control on no-go trials in the group condition were required, the No-go P3 amplitude should be more pronounced in the group condition compared to the individual condition.

\section{METHODS}

\section{Participants}

Twenty participants (5 men and 15 women; age, 1927 years) took part in the experiment. All were righthanded and had normal or corrected-to-normal vision. They received payment for their participation.

\section{Materials}

\section{Stimuli}

Digital photographs of a human hand pointing to the right or to the left were presented as stimuli. The ring on the index finger of the hand was either red or green 
ring (cf., Figure 2). The stimuli were presented centrally, and the ring always appeared at the same location. The picture size was about $22 \times 17$ visual degrees horizontally and vertically. The ring size was approximately 1.2 visual degrees horizontally and vertically, and the size of the pointing finger was approximately 1.7 visual degrees horizontally and vertically. The stimuli were presented on a 22-in. VGA monitor (Iiyama Vision Master Pro 510) using a PC system equipped with a VSG2/5 graphic accelerator (Cambridge Research Systems, Rochester, U.K.). Presentation and response collection were realized through custom-made software.

\section{Response Collection}

Participants pressed single response buttons connected to the PC that presented the stimuli.

\section{Electrophysiological Recording}

Electrophysiological data were recorded from 30 scalp electrodes per head. Additionally, bipolar recordings of vertical and horizontal electro-oculograms (EOGs) were made from electrodes above and below the left eye, as well as from electrodes at the outer canthi of both eyes. In the group condition, the electrodes from each head were connected to a separate amplifier. EEG and EOG were recorded continuously throughout the experimental session, sampled at $500 \mathrm{~Hz}$, and digitally filtered between direct current and $200 \mathrm{~Hz}$. All EEG recordings were referenced to an electrode affixed to the nose. Continuous data were then segmented to the onset of the stimulus presentation. These single-trial data were corrected for eye movements (Gratton, Coles, \& Donchin, 1983) and edited with a software algorithmic artifact rejection program. Only artifact-free trials were averaged to create the ERP separately for each condition and for each subject. For analyses and presentation, data were lowpass-filtered at $8 \mathrm{~Hz}(12 \mathrm{~dB} / \mathrm{oct})$.

\section{Procedure and Design}

Each trial began with a fixation cross presented for 500 msec. It was followed by presentation of one of the stimuli. Participants performed a go/no-go task. They were instructed to respond to red or green by pressing the response button placed in front of them as fast as possible. The direction in which the finger pointed was irrelevant to the response. On half of the trials, the irrelevant pointing direction corresponded to the response to be given (compatible trials). On the other half of trials, the pointing direction referred to the action that was not to be performed (incompatible trials). Each participant performed the go/no-go task alone (individual condition) and alongside another agent responding to complementary color (group condition). Half of the participants started with the individual condition, and half started with the group condition. In the group condition, participants sat next to each other with a distance of about $40 \mathrm{~cm}$ between them. In the individual condition, the chair next to them remained empty. In each condition, participants responded with the right index finger on two blocks and with the left index finger on the other two blocks. In the group condition, both participants always used the same hand at a time (either left or right).

Participants completed 4 blocks of 100 trials in each condition. On 50 of these trials, the stimulus appeared right after fixation. Only these trials are reported in the present article. On the other half of trials (randomly intermixed), a picture of the hand pointing to the left or to the right with a gray ring on the index finger was shown. After $500 \mathrm{msec}$, the color of the ring turned red or green. During this observation interval, participants could not plan an action because they did not know whose turn it would be. We included this observation interval in the design of our study to investigate the possibility that the social setting affects perceptual or attentional processing of stimuli. ERP analyses of N100 and P100 did not show any significant effects of setting and are thus not further reported here.

\section{RESULTS}

\section{Action Planning: RTs}

Figure 3 shows the RT data for the individual condition and the group condition. A $2 \times 2$ within-subjects analysis of variance (ANOVA) with the factors Condition (individual and group) and Compatibility (compatible and incompatible) was conducted to test the prediction that

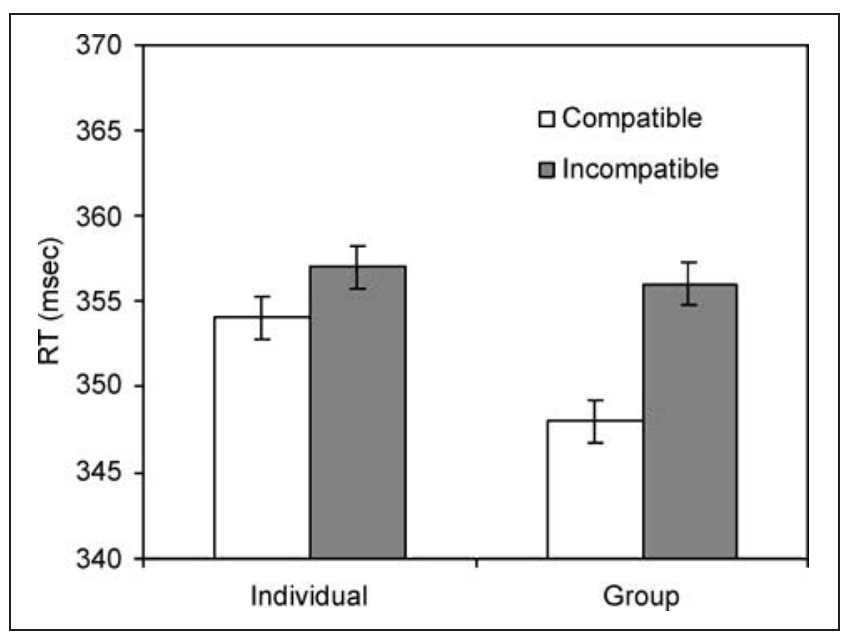

Figure 3. Mean RT on compatible and incompatible trials in individual and group conditions. Confidence interval was calculated based on mean square error following Loftus and Masson (1994). 
a significant compatibility effect would be present in the group, but not in the individual condition. The main effect for Compatibility was significant, $F(1,19)=25.47$, $p<.001$. As predicted, there was a significant interaction between Condition and Compatibility, $F(1,19)=$ $5.57, p<.05$. In the group condition, RTs were significantly slower on incompatible trials than on compatible trials. In the individual condition, there was no significant difference between compatible and incompatible trials.

\section{Action Planning: Go P3}

Figure 4A shows the Go P3 amplitude, locked to stimulus onset. We tested for effects of compatibility and social context as the mean amplitude between 320 and $420 \mathrm{msec}$, around the parietal peak of P3 ( $\pm 50 \mathrm{msec})$ (see Table 1a). Amplitudes were measured in a representative $3 \times 3$ amplitude grid at frontal, central, and parietal leads to test for scalp distribution of this component. A $2 \times 3 \times 3 \times 2$ within-subjects ANOVA, with the factors Condition (group and individual), Anterior/Posterior (frontal, central, and parietal electrodes), Laterality (left, center, and right electrodes), and Compatibility (compatible and incompatible), was conducted. The $\mathrm{P} 3$ amplitude showed a posterior maximum, $F(2,38)=60.40, p<.001$, which is in line with the well-established finding of a $\mathrm{P} 3$ maximum at centroparietal scalp sites (e.g., Tekok-Kilic et al., 2001; Polich, 1993). As predicted based on previous findings, the P3 amplitude was larger on compatible trials, where the pointing direction corresponded to the action to be performed, $F(1,19)=9.72, p<.01$. This effect was most pronounced at central and parietal sites, Anterior/ Posterior $\times$ Compatibility, $F(2,38)=8.23, p<.01$, and was more pronounced toward the left hemisphere, Laterality $\times$ Compatibility, $F(2,38)=7.03, p<.01$. None of the other effects, including the factor condition, reached significance, $p>.10$.

Inspection of the grand averages suggests that, in addition to the main effect for compatibility that can be observed around the P3 peak, the predicted difference in the electrophysiological response between incompatible stimuli referring to the coactor's action and incompatible stimuli not referring to an action might also be present. The late portion of the $\mathrm{P} 3$ complex for incompatible stimuli appears larger in the group condition than in the individual condition. This effect starts around the P3 peak, predominantly at the frontal and central electrodes, and increases over time.

To test for this effect, we conducted another $2 \times 3 \times$ $3 \times 2$ within-subjects ANOVA with the same factors as above for the late phase of the P3 complex (mean amplitude, $400-600 \mathrm{msec}$ ) (see Table 1b). Within this time window, a posteriorly pronounced positivity can still be observed, $F(2,38)=34.78, p<.001$. There was a

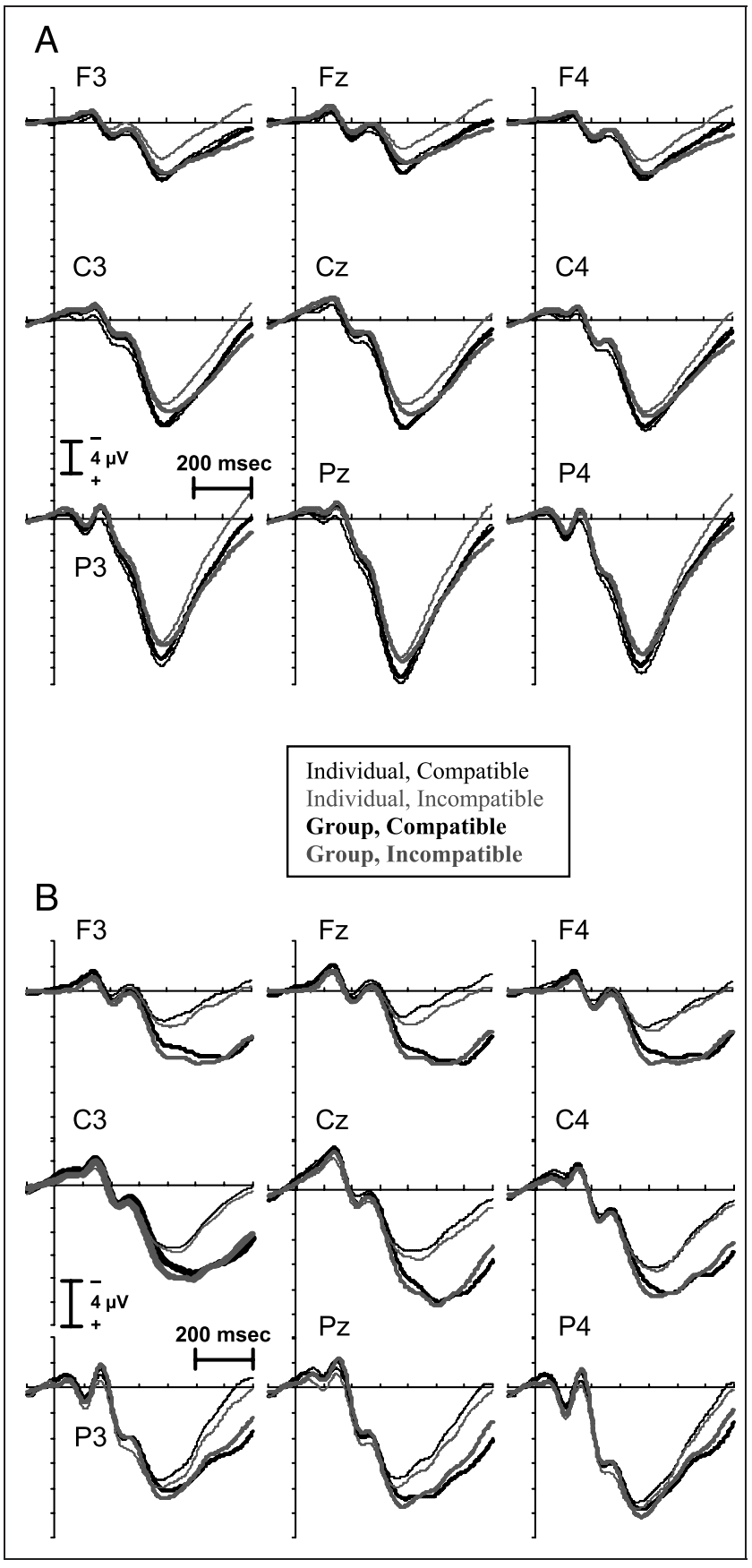

Figure 4. Grand averages showing Go P3 and No-go P3. This figure shows the electrophysiological response to compatible and incompatible trials in the group and individual conditions. (A) Go trials. (B) No-go trials.

significant interaction between Anterior/Posterior and Laterality, $F(1,19)=11.13, p<.001$, but this was not mediated by neither Condition or Compatibility. Importantly, the interaction between Condition and Compatibility was significant, $F(1,19)=9.81, p<.01$. The amplitude was not affected by compatibility in the group condition, $F<1$, but by compatibility in the individual condition, $F(1,19)=8.59, p<.01$. 
Table 1. Electrophysiological Responses (Means and Standard Deviations in Microvolts): (a) on Go Trials (320-420 msec after Stimulus Onset); (b) on Go Trials (400-600 msec after Stimulus Onset); and (c) on No-go Trials (450-550 msec after Stimulus Onset)

\begin{tabular}{|c|c|c|c|c|}
\hline & \multicolumn{2}{|c|}{ Group } & \multicolumn{2}{|c|}{ Individual } \\
\hline & Compatible & Incompatible & Compatible & Incompatible \\
\hline \multicolumn{5}{|l|}{ (a) } \\
\hline F3 & $6.05(4.53)$ & $5.40(4.08)$ & $5.76(4.49)$ & $3.78(5.12)$ \\
\hline $\mathrm{Fz}$ & $4.99(4.68)$ & $4.01(4.21)$ & $4.45(4.84)$ & $2.68(5.47)$ \\
\hline $\mathrm{F} 4$ & $6.02(4.42)$ & $5.52(4.15)$ & $5.74(4.22)$ & $4.16(4.99)$ \\
\hline $\mathrm{C} 3$ & $11.45(6.21)$ & $9.99(6.45)$ & $11.78(7.70)$ & $9.03(7.71)$ \\
\hline $\mathrm{Cz}$ & $11.72(6.81)$ & $9.97(7.28)$ & $11.51(8.42)$ & $9.02(8.64)$ \\
\hline C4 & $11.82(6.22)$ & $10.64(6.45)$ & $12.03(7.45)$ & $9.95(7.47)$ \\
\hline P3 & $16.03(7.09)$ & $14.71(7.86)$ & $16.48(8.47)$ & $13.63(8.55)$ \\
\hline $\mathrm{Pz}$ & $18.29(8.04)$ & $16.59(8.76)$ & $18.61(9.67)$ & $15.61(9.62)$ \\
\hline P4 & $16.97(7.35)$ & $15.82(7.70)$ & $17.59(8.87)$ & $15.26(8.79)$ \\
\hline \multicolumn{5}{|l|}{ (b) } \\
\hline F3 & $4.05(4.82)$ & $4.46(4.19)$ & $3.71(4.11)$ & $1.62(5.14)$ \\
\hline $\mathrm{Fz}$ & $3.15(4.64)$ & $3.38(4.08)$ & $2.83(4.22)$ & $1.05(5.37)$ \\
\hline F4 & $3.87(4.45)$ & $4.51(3.95)$ & $3.66(3.57)$ & $2.21(5.19)$ \\
\hline C3 & $8.44(4.98)$ & $8.68(5.31)$ & $8.78(6.08)$ & $6.28(7.28)$ \\
\hline $\mathrm{Cz}$ & $8.89(5.38)$ & $9.14(5.89)$ & $9.26(6.36)$ & $7.05(8.14)$ \\
\hline $\mathrm{C} 4$ & $8.55(4.36)$ & $8.90(4.73)$ & $8.84(5.09)$ & $7.11(6.96)$ \\
\hline P3 & $9.16(5.55)$ & $9.93(5.93)$ & $9.56(6.76)$ & $7.08(7.68)$ \\
\hline $\mathrm{Pz}$ & $10.79(6.59)$ & 11.21 (6.99) & $11.17(7.12)$ & $8.66(8.30)$ \\
\hline $\mathrm{P} 4$ & $9.01(5.29)$ & $9.66(5.76)$ & $9.52(5.91)$ & $7.66(7.43)$ \\
\hline \multicolumn{5}{|l|}{ (c) } \\
\hline F3 & $4.88(4.45)$ & $5.60(4.85)$ & $1.29(3.39)$ & $1.85(3.94)$ \\
\hline $\mathrm{Fz}$ & $5.34(4.45)$ & $5.68(4.42)$ & $0.81(3.09)$ & $1.65(4.19)$ \\
\hline $\mathrm{F} 4$ & $5.01(4.50)$ & $5.51(4.68)$ & $1.83(3.10)$ & $2.05(3.75)$ \\
\hline C3 & $7.20(5.08)$ & $7.65(5.53)$ & $4.08(3.70)$ & $4.43(4.48)$ \\
\hline $\mathrm{Cz}$ & $8.55(6.10)$ & $8.83(6.23)$ & $4.09(4.19)$ & $4.71(4.68)$ \\
\hline $\mathrm{C} 4$ & $7.62(5.22)$ & $7.79(5.48)$ & $5.10(3.31)$ & $5.17(4.12)$ \\
\hline P3 & $6.58(5.73)$ & $6.52(6.48)$ & $4.54(3.82)$ & $5.36(4.57)$ \\
\hline $\mathrm{Pz}$ & $7.86(6.66)$ & $7.60(7.44)$ & $4.72(4.38)$ & $5.47(4.78)$ \\
\hline P4 & $6.94(5.75)$ & $6.77(6.43)$ & $5.88(3.88)$ & $6.11(4.47)$ \\
\hline
\end{tabular}

To directly compare the two effects observed in the earlier and later time windows, a within-subjects ANOVA was performed only on the group data, with the factors Latency (320-420 vs. 400-600 msec), Anterior/Posterior (frontal, central, and parietal electrodes), Laterality (left, center, and right electrodes), and Compatibility (compatible and incompatible). This analysis indicated differences in the topographical distribution of the two effects. The interaction between Latency, Anterior/Posterior, and Compatibility was significant, $F(2,38)=6.70$ 
$p<.01$. The interaction between Latency, Laterality, and Compatibility was marginally significant, $F(2,38)=2.49$, $p=.09$. The four-way interaction was also significant, $F(4,76)=2.53, p<.05$. The earlier effect, based on a greater amplitude on compatible trials than on incompatible trials, was strongest at the central and parietal electrodes and was lateralized to the left, whereas the later effect, showing no difference in amplitude for compatible and incompatible trials, was of equal size at all sites.

\section{Action Control: No-go P3}

Figure $4 \mathrm{~B}$ shows the grand averages for No-go P3, which is locked to stimulus onset. A $2 \times 3 \times 3 \times 2$ ANOVA with the same factors as in all the analyses above was conducted. The time window selected for the analysis was 450-550 msec, wherein the amplitude effect was maximal (see Table 1c). Overall, this component showed a centroparietal maximum, $F(2,38)=14.99, p<.001$. Of central interest, the amplitude of No-go P3 was larger in the group condition compared to that in the individual condition, $F(1,19)=6.36, p<.05$. This effect was most pronounced at anterior electrodes, Condition $\times$ Anterior/Posterior, $F(2,38)=11.11, p<.001$. Separate analyses for the three electrode lines revealed significant effects of Condition for frontal electrodes, $F(1,19)=$ $10.82, p<.01$, and central electrodes, $F(1,19)=7.68$, $p<.05$, but not for parietal electrodes $(p>.15)$.

\section{DISCUSSION}

The analyses showed (1) a significant compatibility effect for RTs in the group condition, but not in the individual condition; (2) a larger P3 amplitude peak on compatible vs. incompatible go trials in both conditions; (3) a much smaller positivity in the late portion of the P3 complex for incompatible go trials in the individual condition vs. incompatible go trials in the group condition; and (4) a larger No-go P3 amplitude in the group condition. In the following, we discuss each of these findings.

\section{Action Planning}

Behavioral and electrophysiological analysis of the go trials provided evidence that acting together modulated processes of action planning. RT analysis revealed a significant compatibility effect in the group condition. Responses were faster when the pointing direction corresponded to one's own action and were slower when the pointing stimulus referred to the other's action. This finding can be explained by the assumption that, in a group setting, the irrelevant pointing stimulus automatically activated a representation of one's own or of the other's action, depending on which action it referred to. Thus, on compatible trials, one's own action was activated not only through the relevant color cue, but also through the irrelevant pointing direction, leading to facilitation. On incompatible trials, a conflict between two competing actions had to be resolved, causing longer RTs. This RT pattern supports the assumption that one's own action and the other's action were coded in a common representational format. No such compatibility effect was observed in the individual setting because only one's own action alternative was represented. Whether the compatibility effect in the group is due to facilitation on compatible trials or due to inhibition on incompatible trials cannot be determined based on the RT analyses. One must be cautious in comparing numerical differences between the two settings, as the overall level of RTs in the two conditions could differ.

In the individual and in the group condition, the Go P3 amplitude at central and parietal sites was larger on trials where the pointing direction corresponded to the action to be performed. Similar findings have been obtained in studies using two-choice RT tasks, where a reduced P3 amplitude peak on incompatible trials was observed over parietal and central regions (Zhou et al., 2004; Valle Inclán, 1996; Renault et al., 1989; Ragot, 1984). Given that there was no behavioral compatibility effect in the individual condition, it seems unlikely that the reduced P3 amplitude peak on incompatible trials reflects a response selection conflict. Rather, we suggest that this effect is a consequence of perceptual interference (cf., Valle Inclán, 1996).

However, we also found evidence for the claim that another's action was represented in a way similar to one's own. At frontal sites, incompatible stimuli evoked a larger $\mathrm{P} 3$ in the group condition than in the individual condition. At more posterior sites, a larger positivity in the group condition was observed after the P3 peak. Thus, stimuli referring to the other's action alternative seemed to be processed in a way similar to that when referring to one's own action. This can be explained by the assumption that the two action alternatives were represented in a functionally equivalent way. Both when the pointing stimulus corresponded to the action at one's own disposal and when it corresponded to the action at the other's disposal was a relation between the pointing stimulus and an action processed. In contrast, when the stimulus did not refer to an action, as on incompatible trials in the individual condition, no such relation was processed.

It is difficult to determine the exact nature of this effect because, at frontal sites, it appears to be part of a P3, whereas at more posterior sites, it occurs later in the course of processing. It has been suggested that the frontal P3 and the parietal P3 are generated by different neural sources (cf., e.g., Verbaten, Huyben, \& Kemner, 1997). This creates the possibility that the observed 
effect is a separate $\mathrm{P} 3$ effect that has its origin at frontal regions and then spreads to more posterior sites. It could reflect a stimulus evaluation process whereby a stimulus referring to the coactor's action is given a meaning in terms of action relevance similar to that when referring to one's own action. Further studies that allow for localization better than that of the EEG are needed to determine the cortical origin for this anteriorization. At present, we would just like to conclude that two functionally dissociable effects were present. The greater positivity for compatible trials compared to incompatible trials occurred independent of the social context and most likely reflected perceptual interference. In contrast, the greater positivity in response to stimuli referring to the other's action indicates that, in the group condition, a representation of the other's action was formed.

\section{Action Control: No-go P3}

The No-go P3 findings provide clear evidence that response inhibition was stronger in the group condition compared to the individual condition. This difference was most pronounced at frontal and central electrodes, and was diminished at more posterior sites. This finding is in line with the reported anteriorization of the No-go P3 in tasks requiring inhibition (cf., e.g., Falkenstein et al., 1995). The stronger tendency for action inhibition in the group can be explained by the assumption that perceiving a stimulus that is action-relevant for the other leads to the activation of representational structures involved in one's own execution of this action. Increased response inhibition was required to prevent one from responding on no-go trials in the group.

A recent ERP study by van Schie, Mars, Coles, and Bekkering (2004) supports this interpretation. This study demonstrated that a lateralized readiness potential develops when one anticipates somebody else's action, just as if one were to perform the action oneself. This leads to the question of the lead of which inhibitory processes operate in a social action context?" They could be restricted to the level of action planning, but could also occur at the level of movement execution. Two pieces of evidence suggest that an inhibitory mechanism operates mainly at the action planning level. First, the No-go P3 has been interpreted as reflecting processes of action control at the planning level. For example, in a study by Pfefferbaum et al. (1985), the No-go P3 appeared not only when the execution of a button press had to be inhibited, but also when one had to refrain from silent counting. Second, recordings of electromyogram in the study by van Schie et al. also suggest that covert response activation following anticipation of somebody else's action does not extend to the periphery. Nevertheless, we do not want to exclude the possibility that, in the group condition, inhibition also took place at the level of motor control.
To summarize, our findings provide evidence that acting together poses special demands on processes related to action planning and control. The results indicate that these special demands emerge because a representation of the other's action is formed. They suggest that one's own actions and others' actions are represented in a common representational domain, so that observing or anticipating somebody else's action also activates one's own action representation. In particular, three different findings support this claim. First, Go P3 findings show that action-relevant stimuli referring to the partner's action elicited an electrophysiological response similar to that when referring to one's own action. Second, the pattern of RTs suggests that a representation of the other's action interacted with a representation of one's own action during action planning. Finally, No-go P3 findings demonstrate that anticipating the other's action caused a tendency to act, which had to be suppressed.

The present ERP data cannot be taken to make any claims about localization. However, based on previous results, we would like to speculate on brain systems that are involved in the processes of action planning and control in the group condition. First, we consider what happens when it is one's own turn but the stimulus refers to the other's action (go, incompatible). We assume that, in this case, two competing action representations are activated. Many studies have shown that the human mirror system is activated during the observation and imagination of another's actions (see Introduction). Thus, it seems most likely that seeing a stimulus that refers to the other's action activates a corresponding representation in one's own action system. It seems possible that the resulting conflict of action selection is resolved outside of the mirror system, supported by areas involved in action monitoring, conflict detection, and action selection, such as the anterior cingulate cortex (cf., e.g., Botvinick et al., 2004; Luu \& Pederson, 2004) and the dorsolateral prefrontal cortex (cf., e.g., Ridderinkhof, van den Wildenberg, Segalowitz, \& Carter, 2004).

Second, consider what happens when the relevant stimulus feature requires a response from the coactor (no-go trials). It has been shown in monkeys (Cisek \& Kalaska, 2004) and humans (Ramnani \& Miall, 2004) that the dorsal premotor cortex is activated when individuals respond to stimuli based on a previously acquired stimulus-response mapping. Is this area also activated when one sees a stimulus that requires another individual's action? Although this seems to be the case in monkeys (Cisek \& Kalaska, 2004), in humans, the ventral premotor cortex, which forms part of the mirror system, and areas related to mental state attribution (paracingulate cortex and superior temporal cortex), have been shown to be activated in such situations (Ramnani \& Miall, 2004). Thus, at least based on the findings of Ramnani and Miall (2004), it seems likely that, in our study, the mirror system was activated during no-go trials. 
Assuming that observing or imagining somebody else's action always activates one's own motor representation of this action to some degree, one may wonder why we do not copy others' actions all the time. Our No-go P3 finding suggests that an inhibitory mechanism is responsible for suppressing action tendencies resulting from the perception of stimuli requiring another's action. This inhibitory mechanism seems to be impaired in patients with frontal lobe lesions, who have difficulty refraining from imitating actions they have seen (Brass, Derrfuss, Matthes-von Cramon, \& von Cramon, 2003). In line with these findings, a recent functional magnetic resonance imaging study by Brass, Derrfuss, and von Cramon (2005) suggests that a specific set of brain areas is involved in the inhibition of actions that have been preactivated through action observation. The authors found activation in the anterior frontomedian cortex and the temporoparietal junction-areas that are typically involved in perspective taking and determining agency (cf., Ruby \& Decety, 2001). They suggest that the inhibition of imitative response tendencies recruits brain mechanisms that support the distinction between selfgenerated and externally generated actions. Whether this also holds for situations in which individuals act in turns remains to be investigated.

The present study is a first step toward studying realtime dyadic interactions with neuroscientific methods (for other studies taking a similar approach to capture the mutual influences of two minds on another, see e.g., Decety, Jackson, Sommerville, Chaminade, \& Meltzoff, 2004; Ramnani \& Miall, 2004; Singer et al., 2004; van Schie et al., 2004; Rilling et al., 2002; McCabe, Houser, Ryan, Smith, \& Trouard, 2001). Our findings contribute to the understanding of how shared representations of actions and tasks emerge. Such shared representations could provide the basis for joint action, where two or more individuals coordinate their actions in order to achieve common goals (cf., Knoblich \& Jordan, 2003; Sebanz, Bekkering, \& Knoblich, 2006). The cognitive and neuronal processes underlying these more complex forms of interindividual coordination remain to be addressed in future studies.

\section{Acknowledgments}

The study reported in this article was conducted at the Department of Psychology, Max Planck Institute for Human Cognitive and Brain Sciences. We thank Renate Tschackert and Birte Kröger for their help in data collection.

Reprint requests should be sent to Natalie Sebanz, Psychology Department, Rutgers University, 101 Warren Street, Newark, NJ 07102, USA, or via e-mail: sebanz@psychology.rutgers.edu.

\section{REFERENCES}

Altschuler, E. L. (2000). M $\mu$ wave blocking by observation of movement and its possible use as a tool to study theory of other minds. Social Neuroscience, 26, 68.
Blakemore, S.-J., \& Decety, J. (2001). From the perception of action to the understanding of intention. Nature Reviews Neuroscience, 2, 561-567.

Bokura, H., Yamaguchi, S., \& Kobayashi, S. (2001). Electrophysiological correlates for response inhibition in a Go/NoGo task. Clinical Neurophysiology, 112, 2224-2232.

Botvinick, M., Braver, T. S., Yeung, N., Ullsperger, M., Carter, C. S, \& Cohen, J. D. (2004). Conflict monitoring: Computational and empirical studies. In M. I. Posner (Ed.), Cognitive neuroscience of attention (pp. 91-102). New York: Guilford Press.

Brass, M., Bekkering, H., \& Prinz, W. (2001). Movement observation affects movement execution in a simple response task. Acta Psychologica, 106, 3-22.

Brass, M., Derrfuss, J., Matthes-von Cramon, G., \& von Cramon, D. Y. (2003). Imitative response tendencies in patients with frontal lesions. Neuropsychology, 17, 265-271.

Brass, M., Derrfuss, J., \& von Cramon, D. Y. (2005). The inhibition of imitative and overlearned responses: A functional double dissociation. Neuropsychologia, 43, 89-98.

Buccino, G., Binkofski, F., \& Riggio, L. (2004). The mirror neuron system and action recognition. Brain and Language, 89, 370-376.

Chartrand, T. L., \& Bargh, J. A. (1999). The chameleon effect: The perception-behavior link and social interaction. Journal of Personality and Social Psychology, 76, 893-910.

Cisek, P., \& Kalaska, J. F. (2004). Neural correlates of mental rehearsal in dorsal premotor cortex. Nature, 431, 993-996.

Cochin, S., Barthelemy, C., Roux, S., \& Martineau, J. (1999). Observation and execution of movement: Similarities demonstrated by quantified electroencephalography. European Journal of Neuroscience, 11, 1839-1842.

Decety, J., Grèzes, J., Costes, N., Perani, D., Jeannerod, M., Procyk, E., Grassi, F., \& Fazio, F. (1997). Brain activity during observation of actions. Influence of action content and subject's strategy. Brain, 120, 1763-1777.

Decety, J., Jackson, P. L., Sommerville, J. A., Chaminade, T., \& Meltzoff, A. N. (2004). The neural bases of cooperation and competition: An fMRI investigation. Neuroimage, 23, 744-751.

Falkenstein, M., Koshlykova, N. A., Kiroj, V. N., Hoormann, J., \& Hohnsbein, J. (1995). Late ERP components in visual and auditory Go/NoGo tasks. Electroencephalography and Clinical Neurophysiology: Evoked Potentials, 96, 36-43.

Fallgatter, A. J., \& Strik, W. K. (1999). The NoGo-anteriorization as a neurophysiological standard-index for cognitive response control. International Journal of Psychophysiology, 32, 233-238.

Gallese, V., Fadiga, L., Fogassi, L., \& Rizzolatti, G. (1996). Action recognition in the premotor cortex. Brain, 119, 593-609.

Gastaut, H., \& Bert, J. (1954). EEG changes during cinematographic presentation. Electroencephalography in Clinical Neurophysiology, 6, 433-444.

Grafton, S. T., Arbib, M. A., Fadiga, L., \& Rizzolatti, G. (1996). Localization of grasp representations in humans by positron emission tomography: 2. Observation compared with imagination. Experimental Brain Research, 112, 103-111.

Gratton, G., Coles, M. G. H., \& Donchin, E. (1983). A new method for off-line removal of ocular artifact. Electroencephalography and Clinical Neuropsychology, 55, 468-484. 
Greenwald, A. G. (1970). Sensory feedback mechanisms in performance control: With special reference to the ideo-motor mechanism. Psychological Review, 77, 73-99.

Grèzes, J., Armony, J. L., Rowe, J., \& Passingham, R. E. (2003). Activations related to "mirror" and "canonical" neurons in the human brain: An fMRI study. Neuroimage, 18, 928-937.

Grèzes, J., \& Decety, J. (2001). Functional anatomy of execution, mental simulation, observation, and verb generation of actions: A meta-analysis. Human Brain Mapping, 12, 1-19.

Hari, R., Fross, N., Avikainen, E., Kirveskari, E., Salenius, S., \& Rizzolatti, G. (1998). Activation of human primary motor cortex during action observation: A neuromagnetic study. Proceedings of the National Academy of Sciences, U.S.A., 95, 15061-15065.

Hommel, B. (1996). Toward an action-concept model of stimulus-response compatibility. In B. Hommel \& W. Prinz (Eds.), Theoretical issues in stimulus-response compatibility (pp. 281-320). Amsterdam: North-Holland.

Hommel, B. (2006). How we do what we want: A neuro-cognitive perspective on human action planning. In R. J. Jorna, W. van Wezel, \& A. Meystel (Eds.), Planning in intelligent systems: Aspects, motivations and methods. New York: John Wiley and Sons.

Jackson, S. R., Jackson, G. M., \& Roberts, M. (1999). The selection and suppression of action: ERP correlates of executive control in humans. NeuroReport, 10, 861-865.

Jeannerod, M. (1999). The 25th Bartlett Lecture: To act or not to act: Perspectives on the representation of actions. Quarterly Journal of Experimental Psychology, Human Experimental Psychology, 52A, 1-29.

Kilner, J. M., Paulignan, Y., \& Blakemore, S. (2003). An interference effect of observed biological movement on action. Current Biology, 13, 522-525.

Knoblich, G., \& Jordan, S. (2003). Action coordination in groups and individuals: Learning anticipatory control. Journal of Experimental Psychology: Learning, Memory, and Cognition, 29, 1006-1016.

Kohler, E., Keysers, C., Umilta, M. A., Fogassi, L., Gallese, V., \& Rizzolatti, G. (2002). Hearing sounds, understanding actions: Action representation in mirror neurons Science, 297, 846-848.

Kok, A. (2001). On the utility of $\mathrm{P} 3$ amplitude as a measure of processing capacity. Psychophysiology, 38, 557-577.

Kornblum, S., Hasbroucq, T., \& Osman, A. (1990). Dimensional overlap: Cognitive basis for stimulus-response compatibility: A model and taxonomy. Psychological Review, 97, 253-270

Loftus, G. R., \& Masson, M. E. J. (1994). Using confidence intervals in within-subjects designs. Psychonomic Bulletin and Review, 1, 476-490.

Luu, P., \& Pederson, S. M. (2004). The anterior cingulate cortex: Regulating actions in context. In M. Posner (Ed.), Cognitive neuroscience of attention (pp. 232-242). New York: Guilford Press.

McCabe, K., Houser, D., Ryan, L., Smith, V., \& Trouard, T. (2001). A functional imaging study of cooperation in two-person reciprocal exchange. Proceedings of the National Academy of Sciences, U.S.A., 98, 11832-11835.

Pfefferbaum, A., Ford, J. M., Weller, B. J., \& Kopell, B. S. (1985). ERPs to response production and inhibition. Electroencephalography and Clinical Neurophysiology, 60, 423-434.
Polich, J. (1993). P300 in clinical applications: Meaning, method, and measurement. In E. Niedermeyer \& F. Lopes da Silva (Eds.), Electroencephalography: Basic principles, clinical applications, and related fields (3rd ed., pp. 1005-1018). Baltimore: Williams and Wilkins.

Prinz, W. (1997). Perception and action planning. European Journal of Cognitive Psychology, 9, 129-154.

Ragot, R. (1984). Perceptual and motor space representation: An event-related potential study. Psychophysiology, 2, 159-170.

Ramnani, N., \& Miall, R. C. (2004). A system in the human brain for predicting the actions of others. Nature Neuroscience, 7, 85-90.

Renault, B., Fiori, N., \& Giami, S. (1989). Latencies of event-related potentials as a tool for studying motor processing organization. In B. Renault, M. Kutas, M. G. H. Coles, \& A. W. K. Gaillard (Eds.), Event-related potential investigation of cognition (pp. 217-230). Amsterdam: North-Holland.

Ridderinkhof, K. R., van den Wildenberg, W. P. M., Segalowitz, S., J., \& Carter, C. S. (2004). Neurocognitive mechanisms of cognitive control: The role of prefrontal cortex in action selection, response inhibition, performance monitoring, and reward-based learning. Brain and Cognition, 56, 129-140.

Rilling, J., Gutman, D., Zeh, T., Pagnoni, G., Berns, G., \& Kilts, C. (2002). A neural basis for social cooperation. Neuron, 35, 395-405.

Rizzolatti, G., \& Craighero, L. (2004). The mirror neuron system. Annual Review of Neuroscience, 27, 169-192.

Rizzolatti, G., Fadiga, L., Gallese, V., \& Fogassi, L. (1996). Premotor cortex and the recognition of motor actions Cognitive Brain Research, 3, 131-141.

Rizzolatti, G., Fadiga, L., Matelli, M., Bettinardi, V., Paulesu, E., Perani, D., \& Fazio, F. (1996). Localization of grasp representations in humans by PET: 1 . Observation versus execution. Experimental Brain Research, 111, 246-252.

Rizzolatti, G., Fogassi, L., \& Gallese, V. (2001). Neurophysiological mechanisms underlying the understanding and imitation of action. Nature Reviews Neuroscience, 2, 661-670.

Roberts, L. E., Rau, H., Lutzenberger, W., \& Birbaumer, N. (1994). Mapping P300 waves onto inhibition: Go/No-Go discrimination. Electroencephalography and Clinical Neurophysiology, 92, 44-55.

Ruby, P., \& Decety, J. (2001). Effect of subjective perspective taking during simulation of action: A PET investigation of agency. Nature Neuroscience, 4, 546-550.

Sebanz, N., Bekkering, H., \& Knoblich, G. (2006). Joint actions: Bodies and minds moving together. Trends in Cognitive Sciences, 10, 70-76.

Sebanz, N., Knoblich, G., \& Prinz, W. (2003). Representing others' actions: Just like one's own? Cognition, 88, B11-B21.

Sebanz, N., Knoblich, G., \& Prinz, W. (2005). How two share a task. Journal of Experimental Psychology: Human Perception and Performance, 31, 1234-1246.

Sebanz, N., Knoblich, G., Stumpf, L., \& Prinz, W. (2005). Far from action blind: Representation of others' actions in individuals with autism. Journal of Cognitive Neuropsychology, 22, 433-454.

Simon, J. R. (1990). The effects of an irrelevant directional cue on human information processing. In R. W. Proctor \& T. G. Reeve (Eds.), Stimulus-response compatibility: An integrated perspective (pp. 31-86). Amsterdam: North-Holland. 
Singer, T., Seymour, B., O’Doherty, J., Kaube, H., Dolan, R. J., \& Frith, C. D. (2004). Empathy for pain involves the affective but not sensory components of pain. Science, 303, 1157-1162.

Stürmer, B., Aschersleben, G., \& Prinz, W. (2000). Correspondence effects with manual gestures and postures: A study of imitation. Journal of Experimental Psychology: Human Perception and Performance, 26, 1746-1759.

Tekok-Kilic, A., Shucard, J. L., \& Shucard, D. W. (2001). Stimulus modality and go/nogo effects on P3 during parallel visual and auditory continuous performance tasks. Psychophysiology, 38, 578-589.

Umiltà, M. A., Kohler, E., Gallese, V., Fogassi, L., Fadiga, L., Keysers, C., \& Rizzolatti, G. (2001). I know what you are doing: A neurophysiological study. Neuron, 31, $155-165$.
Valle Inclán, F. (1996). The locus of interference in the Simon effect: An ERP study. Biological Psychology, 43, $147-162$.

van Schie, H. T., Mars, R. B., Coles, M. G. H., \& Bekkering, H. (2004). Modulation of activity in medial frontal and motor cortices during error observation. Nature Neuroscience, 7 , 549-554.

Verbaten, M. N., Huyben, M. A., \& Kemner, C. (1997). Processing capacity and the frontal P3. International Journal of Psychophysiology, 25, 237-248.

Wascher, E., Reinhard, M., Wauschkuhn, B., \& Verleger, R. (1999). Spatial S-R compatibility with centrally presented stimuli: An event-related asymmetry study on dimensional overlap. Journal of Cognitive Neuroscience, 11, 214-229.

Zhou, B., Zhang, J. X., Han, L. H., \& Tan, S. (2004). Spatial congruence in working memory: An ERP study.

NeuroReport, 15, 2795-2799. 